\title{
Regulation of FtsZ levels in Escherichia coli in slow growth conditions
}

\author{
Jaana Männik ${ }^{a}$, Bryant E. Walker ${ }^{b}$ and Jaan Männika,b \\ ${ }^{a}$ Department of Biochemistry \& Cellular and Molecular Biology, ${ }^{b}$ Department of Physics and \\ Astronomy, University of Tennessee, Knoxville, TN 37996, USA
}

Corresponding author: Jaan Männik; JMannik@utk.edu; phone: +1 (865) 9746018

fax: +1 (865) 9747843 
1

2

3

4

5

6

7

8

\section{Abstract}

A key regulator of cell division in most walled bacteria is the FtsZ protein that assembles into protofilaments attached to the membrane at midcell. These dynamic protofilament assemblies, known as the Z-ring, act as a scaffold for more than two dozen proteins involved in synthesis of septal cell envelopes. What triggers the formation of the Z-ring during the cell cycle is poorly understood. In Escherichia coli model organism, the common view is that FtsZ concentration is constant throughout its doubling time and therefore regulation of assembly should be controlled by some yet to be identified protein-protein interactions. Here we show using quantitative analysis of newly developed fluorescent reporter that FtsZ concentration varies in a cell-cycle dependent manner in slow growth conditions and that upregulation of FtsZ synthesis correlates with the formation of the Z-ring. About 4-fold upregulation of FtsZ synthesis in the first half of the cell cycle is followed by its rapid degradation by ClpXP protease in the last $10 \%$ of the cell cycle. The initiation of rapid degradation coincides with dissociation of FtsZ from the septum. Altogether, our data indicate that the Z-ring formation in slow growth conditions in E. coli is controlled by a regulatory sequence where upregulation of an essential cell cycle factor is followed by its degradation.

Keywords: cell division, cell cycle regulation, FtsZ, ClpXP protease

\section{Significance}

FtsZ is the key regulator for bacterial cell division. It initiates division by forming a dynamic ring-like structure, the Z-ring, at the mid-cell. Here we show that, contrarily to the current paradigm, FtsZ concentration in Escherichia coli model organism varies throughout cell cycle in slow growth conditions.

Faster FtsZ synthesis in the first half of the cell cycle is followed by its rapid degradation by ClpXP protease in the end of the cell cycle. Upregulation of FtsZ synthesis correlates with the formation of the Z-ring. Our data demonstrates that in slow growth $E$. coli cell division progresses according to paradigm where upregulation of essential cell cycle factor is followed by its degradation. 


\section{Introduction}

25

26

27

Cell division in E. coli and other bacteria is an essential process subject to spatial and temporal coordination with the chromosome replication cycle $(1,2)$. The coordination guarantees with high fidelity that bacteria in steady-state growth divide once per initiation of chromosome replication, and that division occurs between completely replicated chromosomes. Significant progress has been made in identifying and unravelling the molecular mechanism involved in correct positioning of the bacterial cell division machinery, the divisome, relative to the chromosomes. Currently, three partially redundant molecular systems are known in E. coli that are involved in the positioning of its divisome (3). At the same time it has remained largely unclear how the division process is controlled temporally.

The earliest defined event in bacterial cytokinesis is assembly of the tubulin-like FtsZ protein into a dynamic ring-like polymer structure, the Z-ring, which localizes at midcell $(4,5)$. FtsZ is a GTPase that is conserved in nearly all walled bacteria (6). In the presence of GTP FtsZ monomers polymerize into dynamic protofilaments $(7,8)$. It has recently been shown that these filaments treadmill $(9-11)$. The protofilaments form higher order structures such as bundles and sheets in vitro $(12,13)$ and likely also in vivo (14). In $E$. coli protofilaments are tethered to the inner membrane via FtsA (15) and ZipA (16) linkers. These two proteins, together with FtsZ and non-essential Z-ring associated proteins ZapA-D, form an early divisome complex, the Z-ring (5). After formation of the Z-ring, but with a distinct delay (17), about 30 other proteins are recruited to the division site in a specific order forming a mature divisome $(18,19)$. Once divisome has matured, it then carries out synthesis of septal cell wall and membranes (20) and safeguards DNA partitioning between daughter cells $(21,22)$. FtsZ acts as a central scaffold in recruiting and maintaining the Z-ring throughout cell division except the latest stages of constriction (5). Various intrinsic and environmentally generated signals couple to the FtsZ scaffold and lead to its depolymerization in unfavorable conditions. 
47 Currently, it is not clear what triggers formation of the Z-ring. According to the common view, FtsZ

48 concentration during the cell cycle remains constant in both $E$. coli $(1,23,24)$ and in distantly related $B$.

49 subtilis (25). At the same time, the cellular concentration of FtsZ (6-7 $\mu \mathrm{M})$ is much higher than the

50 concentration needed for assembly of FtsZ protofilaments in vitro $(1 \mu \mathrm{M})(8)$. This has led to a paradigm

51 that assembly of the Z-ring is controlled at the level of its assembly $(23,26)$ meaning that not protein

52 levels but their interactions inhibit the Z-ring formation despite high concentration of FtsZ. Changes in

53 these interactions reflecting possibly DNA replication status (27) may trigger the formation of the Z-ring.

54 Spatial regulators, such as SImA mediated nucleoid occlusion (28) and the Ter linkage (29) in E. coli and

55 Noc protein in B. subtilis (30) could potentially couple DNA replication cycle and Z-ring (31) although

56 experimental evidence to support this idea is lacking.

57

Even though a constant FtsZ concentration through the cell-cycle is currently the accepted view,

numerous earlier reports show that FtsZ mRNA levels oscillate during cell cycle in E. coli (32-35). Cell-cycle

dependent variations in the levels of FtsZ have been described also in other bacteria, including

Caulobacter crescentus (36), Prochlorococcus sp. (37), and Synechococcus elongatus (38). It is not clear

62 how the cells could possibly achieve constant levels of protein product from oscillating levels of transcript

63 taken that ribosome levels during the cell cycle remain approximately constant (39). Recently, 15\%

64 variation in FtsZ concentration during the cell cycle was reported in faster growth rates in E. coli (40), but

65 the origin of these variations has remained unclear. Here we show using a recently developed FtsZ

66 fluorescent reporter (41) that FtsZ concentration in E. coli varies more significantly during the cell cycle as

67 the growth rate decreases. We determine that in slow growth conditions the variation in FtsZ levels is

68 achieved by increasing the synthesis rate in the beginning and the degradation rate at the end of the cell 
71 FtsZ correlates with the formation of the Z-ring in slow growth conditions, but it is not a sole factor

72 determining the timing of the Z-ring formation.

\section{Results}

FtsZ concentration varies during cell cycle in slow growth conditions. Cell cycle dependent variations in

fusions have been developed, which can be expressed from the native locus and are the sole copy of FtsZ

78 (41). Here we use one of these constructs, where mNeonGreen (mNG) fluorescent protein is sandwiched

between residues 55 and 56 of FtsZ (FtsZ-mNG), to determine the changes of FtsZ numbers and concentration during the cell cycle (detailed description of all strains is in SI Table S1). For measurements

81 of cells in steady state conditions we grew them in microfluidic mother machine channels $(42,43)$. The

82 linear colonies in mother machine channels allow determining spatial distribution of fluorescent intensities from individual cells through the division process without uncertainties introduced by nearby cells. Such uncertainties are present when agarose pads and dishes are used for imaging. As a first step in the analysis we collected intensity profiles from time-lapse measurements to kymographs (Fig. 1A, B). These kymographs here and in later sections are extended beyond the division events to bring more

87 clearly out variations of FtsZ levels during the cytokinesis. We studied cells in slow growth conditions in M9 glycerol medium with doubling time of $T_{d}=160 \pm 30 \mathrm{~min}$ (Fig. 1A) and in faster growth with doubling time of $T_{d}=74 \pm 19$ min (mean \pm s.d.) where M9 glucose was supplemented with casamino acids (Fig. 1B). Note that all measurements were performed at $28^{\circ} \mathrm{C}$. In addition to the strain carrying FtsZ-mNG, we also measured the FtsZ concentration in a control strain that expressed an FtsZ C-terminal fusion to GFP (FtsZ-

92 GFP) under control of lac-promoter from a plasmid (SI Fig. S1) (9, 44). The FtsZ-GFP strain shows 93 comparable doubling times in slow $T_{d}=160 \pm 29 \mathrm{~min}$ and fast $T_{d}=80 \pm 24$ min growth conditions. In the strain 94 with plasmid expressed FtsZ-GFP, one would not expect to observe cell cycle dependent variations in the 
concentration of the fluorescent proteins. Consistent with this expectation, the concentration of

96 fluorescent FtsZ from plasmid expressed FtsZ-GFP varied less than $10 \%$ during the cell cycle in both slow

97 (Fig. 1C) and fast growth conditions (Fig. 1B) in population average measurements. In contrast, we found a $37 \%$ variation of endogenously expressed fluorescent FtsZ-mNG in slow growth conditions. In fast

99 growth conditions, however, the variation of FtsZ concentration was essentially at the level of the control 100 (10\%). The measurements thus show that FtsZ levels in E. coli undergo cell cycle dependent oscillations 101 with an amplitude that depends on growth conditions.

102 In parallel to the concentration we also determined the variation of fluorescent FtsZ during the cell cycle

103 (Fig. 1E, F). Instead of a monotonic increase, the number of these molecules decreased at the end of the 104 cell cycle in slow growth conditions (Fig. 1E). The decrease started at about 0.9Td when fluorescent FtsZ 105 numbers reached 2.2 times higher level than what is present in the beginning of the cell cycle. The decrease was absent in fast growth conditions (Fig. 1F) and in the control strain with plasmid expressed FtsZ in both growth conditions (Fig. 1E-F, SI Fig. S1). While drop in concentration of FtsZ in the end of the cell cycle could have possibly arisen from slowdown of its synthesis at the end of the cell cycle and concurrent dilution of the protein due to cell growth, the decrease in numbers (about 20\%) shows that FtsZ is degraded in the end of the cell cycle. The data furthermore show that the relative amount of FtsZ degraded at the end of cell cycle decreases with the growth rate.

112 Degradation in the end of cell cycle is caused by ClpXP. We next investigated what factors could be 113 responsible for the fast degradation of FtsZ in the end of the cell cycle. Earlier studies have identified that 114 FtsZ is one of the substrates for ClpXP protease in E. coli $(45,46)$. The ClpXP protease consists of hexameric 115 ClpX chaperone that unwinds target proteins by passing them via its central pore in ATP-dependent manner and ClpP peptidase that consists of two heptametric rings (47). The identified ClpX binding sites

117 in FtsZ are localized at its C-terminal domain and in its unstructured linker (48). The earlier studies, 118 however, have not revealed changes in ClpXP activity during the cell cycle (46). To test if ClpXP is 
responsible for the degradation of FtsZ in the end of cell cycle in slow growth conditions, we constructed for degradation of FtsZ in the end of the cell cycle in WT cells.

Synthesis of FtsZ during the cell cycle is not uniform in slow growth conditions. Closer inspection of data from individual $\triangle c l p P$ and $\Delta c l p X$ cells reveals that FtsZ numbers do not increase uniformly in time (Fig. indicates that synthesis of FtsZ is cell cycle dependent in slow growth conditions. In $\triangle c / p P$ and $\Delta c / p X$ cells we can distinguish two periods in the cell cycle that have different FtsZ synthesis rates. These periods correspond to two different slopes in $\mathrm{N}$ vs t curves (Fig. 3B). To find the beginning of these periods and

139 rate of change in FtsZ numbers in each period we performed a piecewise linear fit to the curves from 140 individual cells. Compilation of the fitting results shows (Fig. 3C) a period of upregulated FtsZ synthesis

141 during the cell cycle that starts at time $T_{1}=0.35 \pm 0.2 T_{d}$ and runs until time $T_{2}=0.85 \pm 0.15 T_{d}$ (mean $\pm s$.d.).

142 During this period the synthesis rate is about three times higher than that during the rest of the cell cycle. 
143 Large variations in start and end times of this period among cell population explain why in population

144 averaged data (Fig. 2A) the period with higher synthesis rate did not reveal itself.

145 The times $T_{1}$ and $T_{2}$ cited above did not include corrections for the maturation of the fluorescent reporter.

146 To make this correction we measured maturation time of $\mathrm{mNG}$ in our cells using an approach described

147 earlier (50) (see Materials and Methods). Measurements of WT cells in slow and fast growth conditions,

148 and $\Delta c / p X$ cells in slow growth conditions yielded all maturation half-lifetime of $25 \pm 3$ min corresponding

149 to $(0.15 \pm 0.02) \mathrm{T}_{d}$ (SI Fig. S2). Correcting for the maturation by subtracting maturation half-lifetime from

150 the corresponding times (see SI Modelling, Piecewise Linear Fitting) yields corrected estimates $T_{1, \text { cor }}=$

$151(0.2 \pm 0.2) T_{d}$ for the start and $T_{2, \text { cor }}=(0.7 \pm 0.15) T_{d}$ for the end of the faster synthesis period in $\Delta c / p P$ and

$152 \Delta c / p X$ cells. Fig. 3D summarizes these results in terms of rate of change in FtsZ numbers during the cell

153 cycle.

154 As expected from measurements of $\Delta c / p P$ and $\Delta c l p X$ strains, FtsZ synthesis rates also varied in WT cells

155 during the cell cycle. In addition to upregulation in the synthesis rates, $\mathrm{N}$ vs $\mathrm{t}$ curves of individual cells also

156 had a distinct period in the end of the cell cycle corresponding to rapid degradation of FtsZ (Fig. 3E-H). We

157 included this period in the piecewise linear fits denoting the beginning of the period by $T_{3}$ and its end by

$158 \mathrm{~T}_{4}$ (Fig. 3F, G). Including correction for the maturation time of mNG, FtsZ synthesis was upregulated in WT

159 cells from $T_{1, \text { cor }}=(0.1 \pm 0.12) T_{d}$ to $T_{2, \text { cor }}=(0.6 \pm 0.1) T_{d}$ (mean \pm s.d.). During this period, the rate of FtsZ synthesis

160 was about four times higher than in the beginning of the cell cycle (Fig. $3 \mathrm{H}$ ) similar to the change observed

161 in $\Delta c l p P$ and $\Delta c l p X$ cells. After the $T_{2}$ point, the synthesis of FtsZ slows-down. From $T_{2, \text { cor }}=(0.6 \pm 0.1) T_{d}$ to $T_{3}$

$162=(0.90 \pm 0.04) T_{d}$ FtsZ synthesis rates were comparable to those in the beginning of the cell cycle, i.e. from

1630 to $0.1 \mathrm{~T}_{\mathrm{d}}$. The last period in the cell cycle from $T_{3}=(0.9 \pm 0.04) \mathrm{T}_{\mathrm{d}}$ to $\mathrm{T}_{4}=(1.0 \pm 0.05) \mathrm{T}_{\mathrm{d}}$ showed rapid decrease

164 in FtsZ numbers. This period is clearly visible also in population average curves of Fig. 1E. Since the effect

165 of degradation of the protein is immediate on the fluorescence no corrections were applied to the latter 
two times. The end of the degradation period coincided in good approximation with the end of the cell cycle. Fig. $3 \mathrm{H}$ summarizes the WT results in terms of rate of change in FtsZ numbers during the cell cycle.

It is noticeable that while the beginning $\left(T_{1}\right)$ and end $\left(T_{2}\right)$ of FtsZ synthesis upregulation showed substantial cell-to-cell variability, the beginning $\left(T_{3}\right)$ and end $\left(T_{4}\right)$ of fast degradation of FtsZ showed less stochasticity (Fig. 3G). This indicates that different types of regulation are involved in controlling synthesis and

171 degradation. While synthesis is likely controlled at the transcriptional level and is known to have a high

172 level of stochasticity (51) the same appears not to be the case for degradation.

173 We also attempted the same analysis for WT cells in fast growth conditions. Since degradation of FtsZ was

174 not visible in most individual $\mathrm{N}$ vs t curves, only periods of slow and fast FtsZ synthesis were determined.

175 The analysis indicated about two fold increase in FtsZ synthesis rate from $T_{1, \text { cor }}=0$ to $T_{2, \text { cor }}=0.5 \mathrm{~T}_{\mathrm{d}}$ compared

176 to the remainder of the cell cycle (SI Fig. S3). This difference is statistically significant in t-test $\left(p<10^{-5}\right.$ for

177 equal slopes). However, taken that the maturation time of fluorescent reporter in these growth conditions

178 is about a third of the doubling time, our analysis is expected to significantly underestimate differences in

179 the rates and as such is not directly comparable to the values from slow growth conditions.

Quantification of degradation rates in slow growth conditions. Using data from piecewise linear fits to $N$

181 vs $t$ curves from individual cells we next quantify the distribution of relative amounts of FtsZ degraded in

182 the end of the cell cycle. We find a broad distribution of degraded amounts with an average of $22 \pm 14 \%$

183 (Fig. 4A). This amount is calculated relative to the number of FtsZs at end of the cell cycle. There appears

184 to be no obvious correlation between the FtsZ amount at the end of the division and the amount of FtsZ degraded (SI Fig. S4). On average about the same number of FtsZ molecules are degraded in cells where 
Based on single molecule measurements (52), typical time to degrade a single FtsZ-mNG molecule is about

$10 \mathrm{sec}$. It is likely that this time exceeds the time needed for ClpXP and FtsZ to bind to each other.

In addition to degraded amount, we also determined the time-average degradation rate at the end of the cell cycle (Fig. 4B). We found for the cell population the rate $d_{f}=0.010 \pm 0.05 \mathrm{~min}^{-1}$. This rate is about factor two larger than the one found from bulk measurements in unsynchronized E. coli cultures (46). However, this difference should not be over interpreted because $d_{f}$ depends on the protease concentration in the cell (see SI Modelling). The latter is likely to vary from strain to strain and from one growth condition to another.

We also determined the degradation rate of FtsZ during the remainder of the cell cycle. For that end we 197 inhibited protein synthesis by chloramphenicol and observed decay in fluorescent signal of FtsZ-mNG (Fig. synthesis because of maturation of the fluorescent label (50). To avoid this effect, we determined degradation rates after four mNG maturation half-lifetimes (100 $\mathrm{min}$ ) from administering the drug (Fig. two times smaller $\mathrm{d}_{0}=(0.4 \pm 0.2) \cdot 10^{-3} \mathrm{~min}^{-1}$. The apparent degradation rate of $\Delta c / p X$ strain may reflect values measured in $\Delta c l p X$ strain from the WT one yields a ClpXP related degradation rate constant of $d_{0}=5 \cdot 10^{-4} \mathrm{~min}^{-1}$ in WT cells. There is thus a 20 -fold increase in ClpXP related degradation of FtsZ in the end

207 of the cell cycle compared to basal rate. 
211 when FtsZ begins to dissociate from the Z-ring ( $\left.T_{\text {dis }}\right)$. We defined the latter as a time in the cell cycle when

212 the total amount of FtsZ in the Z-ring starts to decrease (Fig. 5A). The comparison shows that the two

213 times are highly correlated within the cell population ( $R=0.95)$ and essentially co-incident (Fig. 5B). The

214 data also show that the total time it takes to dissociate FtsZ from the Z-ring does not depend whether

215 ClpXP is present or absent (Fig. 5C). These data together indicate that FtsZ degradation does not drive Z-

216 ring dissociation but is a consequence of it. One can furthermore conclude that the increase in the

217 degradation rate is not triggered by an increase in ClpXP numbers but rather by its accessibility to FtsZ.

218 After FtsZ dissociates from the Z-ring it can be expected to be mostly monomeric. To determine if an

219 increase in the numbers of monomeric FtsZ can explain its rapid degradation at the end of the cell cycle,

220 we artificially induced dissociation of the Z-ring to monomeric FtsZ. For this purpose, we transformed the

221 FtsZ-mNG strain with pA3 plasmid carrying inducible SulA (53). SulA binding to FtsZ in one to one

222 stoichiometry lowers effective concentration of FtsZ in the cell so that it is below critical concentration for

223 protofilament formation (53). SulA binding to FtsZ should leave the latter still accessible for ClpXP

224 degradation because SulA does not bind to C-terminal domain of FtsZ, which contains the recognition

225 elements for ClpX binding $(46,48)$. SulA upregulation and subsequent dissociation of the Z-ring, however,

226 did not lead to any detectable degradation of FtsZ (SI Fig. S5A, B). Moreover, SulA upregulation did not

227 change synthesis rate of FtsZ. In cells where SulA was upregulated in early stages of the cell cycle, FtsZ

228 synthesis followed its well-defined pattern (cf. Fig. 3F) despite SulA expression (SI Fig. S5C, D).

229 Consequently, the increase in monomer FtsZ concentration in the cytoplasm during Z-ring dissociation is

230 not the reason for its rapid degradation at the end of the cell cycle.

231 Correlations between FtsZ upregulation and Z-ring formation. Previously presented data (Fig. 3E) show

232 that FtsZ synthesis rate increases 4 -fold shortly after cell birth at $0.1 T_{d}$ in slow growth conditions. We next

233 investigate to what degree this upregulation correlates with the Z-ring formation. To answer this question

234 we determined from kymographs, such as shown in Fig. 5A, the time when the Z-ring forms $\left(T_{z}\right)$. Note that 
in fast growth conditions, in almost all cells (94\%), the Z-ring formed immediately after cell birth (SI Fig S6). However, in slow growth conditions there was a distinct delay between cell birth and the formation of the Z-ring (Fig. 6A). On average the Z-rings formed at $T_{Z}=0.22 T_{d}$ but the times varied considerably between individual cells (s.d. $=0.13 \mathrm{~T}_{\mathrm{d}}$ ). There was a similarly wide distribution in start times of FtsZ synthesis upregulation (s.d. $=0.13 \mathrm{~T}_{d}$, Fig. 6B). These times preceded on average about $0.1 \mathrm{~T}_{d}(16 \mathrm{~min})$ the times of Z-ring formation. At the level of individual cells the two times showed some level of correlation $(R=0.67$; Fig. $6 C)$. In essentially all cells the Z-ring formed after FtsZ synthesis rate had increased. This indicates that upregulation of FtsZ levels is needed for formation of the Z-ring in slow growth conditions. Consistent with this conclusion, Z-rings formed earlier in cells where the amount of FtsZ was higher at cell birth ( $R=-0.43$; Fig. 6D). At the same time the correlation between concentration of FtsZ at cell birth and timing of Z-ring formation were lower ( $R=-0.15$; Fig. $6 E)$ indicating that FtsZ numbers rather than its concentration is the factor controlling the Z-ring formation.

At the time when the Z-ring formed, the amount of FtsZ in the cells (Fig. 6F) and its concentration (Fig. $6 \mathrm{G})$ were about the same, and independent of the time the Z-ring formed ( $R=-0.02 ; R=-0.05$, respectively). These data together show that cells with a low amount of FtsZ at birth synthesize more FtsZ to reach the level needed for Z-ring formation. However, the variation of both the amount of FtsZ and its concentration at the time of the Z-ring formation was large in cell population with a coefficient of variation CV $=20 \%$. Large variation is indicative that FtsZ level is not the sole factor that controls the timing of the Z-ring formation. Altogether we conclude that FtsZ upregulation is necessary for the formation of the Z-ring in slow growth conditions but is not an immediate trigger for it.

\section{Discussion}

We investigated changes in FtsZ numbers and concentration in slow and fast growth conditions in E. coli, and the effects these changes have on Z-ring formation. We found that in fast growth conditions the Z- 
ring formed in essentially all daughter compartments (94\% of cells) concurrent with the dissociation of

259 FtsZ from the constriction of the mother cell. This observation is consistent with previous reports $(8,54)$.

260 Immediate formation of the Z-ring in fast growing cells indicates that there is no specific cell cycle control

261 over this process. In contrast there is a distinct delay between cell birth and formation of the Z-ring in

262 slow growth conditions. Even though the delay has large cell-to-cell variation $\left( \pm 0.13 T_{d}\right)$, its presence

263 indicates that there is a cell cycle based control over the Z-ring formation.

264 Our data shows that regulatory mechanisms controlling Z-ring formation in slow growth conditions

265 includes a faster FtsZ synthesis rate in the first half and its rapid degradation in the last $10 \%$ of the cell

266 cycle. The progression of these cell cycle events is quantitatively summarized in Fig. 7A, B. To further

267 validate this model we compared it to numerically calculated FtsZ numbers and concentrations. The

268 numerical calculation is based on experimentally determined rates including maturation rate of $\mathrm{mNG}$ (see

269 SI Modelling for details). The only unknown parameter in these calculations is the conversion factor

270 between one recorded fluorescent unit and emission from single FtsZ-mNG. Although we have estimated

271 this conversion factor from photobleaching measurements, the uncertainties involved are large.

272 Therefore we compare the experimental data and calculated curves after normalizing both by their

273 maximum values. The normalized model and population average data show a good agreement (Fig. 7C,

274 D). The two deviate from each other most significantly at time $T_{3}$ when the degradation rate of Fts $Z$

275 increases. The sharp cusp in the model is not present in population average data partially because of the

276 spread of degradation start times in the population. It is also plausible that part of the discrepancy is

277 caused by gradual increase in the degradation rate around time $T_{3}$ instead of discontinuous change that

278 we assumed in our analysis and model. Similarly to degradation rate one would also expect that the

279 synthesis rate in individual cells shows a more complicated behavior than discontinuous change between

280 constant levels at times $T_{1}$ and $T_{2}$. It is well-known that individual cell level protein synthesis show bursts 
of activity (55). These details go beyond our simplified model (Fig. 7A,B), which captures a populationaverage behavior on a coarse temporal resolution.

The FtsZ regulation cycle in slow-growing E. coli shows close resemblance to the one in Caulobacter crescentus swarmer cells. In Caulobacter swarmers, FtsZ synthesis is upregulated after transformation of a swarmer to a stalked cell occurs, and it is downregulated in the beginning and end of the cell cycle (56). Also, as we observed in slow growing $E$. coli, FtsZ is degraded in the end of the cell cycle $(56,57)$. In both organisms the degradation occurs via ClpXP based proteolysis, but in the case of Caulobacter ClpAP appears to play also a significant role (57). In our measurements the effect of ClpAP was modest (Fig. 3).

It has been found that upregulation of FtsZ synthesis in swarmer cells coincides with DNA replication period (56). Interestingly, we observe 4-fold upregulation of FtsZ synthesis in slow growing E. coli from $0.1 \mathrm{~T}_{d}$ to $0.6 \mathrm{~T}_{d}$. This period matches well with the period $\left(0.14-0.51 \mathrm{~T}_{d}\right)$ reported for DNA replication in similar growth conditions (58). Increase in FtsZ mRNA levels upon initiation of DNA replication have been reported before in E. coli $(33,35)$. However, other studies have found maximal transcription levels around the middle of the cell cycle (32) or at the time of the division (34). While these different measurements lack yet consensus to support causative link between FtsZ upregulation and initiation of DNA replication

296 in E. coli, a molecular level link between the two processes has been found in C. crescentus (56). This link 297 is realized by the global cell cycle regulator CtrA that represses both the initiation of DNA replication and transcription of FtsZ (59). underlying molecular networks appear different. First, no global cell cycle regulator similar to CtrA is

301 known to exist in E. coli. Second, in Caulobacter there is only a single promoter controlling FtsZ 302 transcription (56) while in E. coli 7 different promoters are responsible for transcription of FtsZ (60). Of the seven the two proximal ones have been reported to lead to cell cycle dependent transcription of FtsZ 
304 (33), but in addition to promotors other regulatory elements such as RNA E cleavage sites, antisense RNA

305 binding to ftsZ mRNA, and competition between ribosome binding sites on long polycistronic RNA could

306 also play a role (60). Further work to understand cell cycle control on transcription FtsZ in E. coli is clearly

307 warranted after nearly a two decade long pause in studies.

308 In addition to mechanisms controlling FtsZ synthesis, our work also raises question about how ClpXP is

309 able to rapidly degrade FtsZ during the end of the cell cycle. The data show that dissociation of the Z-ring

310 triggers degradation of FtsZ. After dissociation from the Z-ring FtsZ may lose specific protective

311 interactions on its conserved C-terminal domain which has one of the two recognition sites for ClpX (46,

312 48). It has been reported before that one of FtsZ membrane linkers, ZipA, is able to protect FtsZ from

313 degradation by ClpXP (61). Furthermore, it is possible that steric hindrance from the inner membrane

314 reduces accessibility of ClpXP to the C-terminal tail of FtsZ and therefore protects it from degradation. In

315 addition to FtsZ residing in the Z-ring, our data also show that monomeric FtsZ in the cytosol is not the

316 preferred substrate for ClpXP. In light of these arguments we hypothesize that possible ClpXP substrates

317 are FtsZ protofilaments or protofilament aggregates either free in the cytosol or partially attached to the

318 membrane. This hypothesis is consistent with previous in vitro studies showing that the degradation rate

319 of a filament or filament bundle is larger than monomeric FtsZ $(46,62)$.

320 To conclude, our data show that concentration of FtsZ oscillates in E. coli in a cell cycle specific manner in

321 slow growth conditions. The lower FtsZ abundance at cell birth guarantees that the Z-ring does not form

322 immediately at the beginning of the cell cycle. However, large variations in FtsZ numbers and

323 concentrations at the time of Z-ring formation within the cell population indicate that some other factors

324 beyond FtsZ can also play a role in triggering the Z-ring formation. Identifying these putative factors and

325 establishing their link to the chromosome replication cycle remains an important avenue for future

326 research. 


\section{Materials and Methods}

Strains and growth conditions. All strains are derivatives of E. coli K-12 (Keio collection strain BW27783).

The strains and plasmids are described in details in SI Table S1. All cells were grown and imaged in M9

minimal medium (Teknova) supplemented with $2 \mathrm{mM}$ magnesium sulfate. In slow growth conditions $0.3 \%$ GFP strain (chloramphenicol, $40 \mathrm{\mu g} / \mathrm{ml}$ ). Cells were grown and imaged at $28{ }^{\circ} \mathrm{C}$.

\section{Microfluidic chip fabrication.}

Soft-lithography of PDMS (polydimethylsiloxane) was used to fabricate microfluidic chips following

previously described procedure (43). Briefly, silicon molds were fabricated combining e-beam and

photolithography, and reactive ion etching. The mold defined dead-end channels, which were $0.8 \mu \mathrm{m}$

wide, $1.1 \mu \mathrm{m}$ high, and $20 \mu \mathrm{m}$ long. PDMS (Sylgard 184, Dow Corning) was poured on passivated mold

from the mold and cut into pieces. Access holes to each piece, corresponding to one microfluidic device,

Preparation and culturing $E$. coli in microfluidic devices. After overnight growth in liquid media, $1 \mu \mathrm{g} / \mathrm{ml}$

347 of bovine serum albumin (BSA) was added to $1.5 \mathrm{ml}$ of overnight cell culture $\left(\mathrm{OD}_{600}>0.4\right)$ and concentrated microfluidic mother machine device. The cells were then allowed to populate the dead-end channels for 
about $1 \mathrm{hr}$. Once these channels were sufficiently populated, tubing was connected to the device, and the

351 flow of fresh M9 medium with BSA $(1 \mu \mathrm{g} / \mathrm{ml})$ was started. The flow was maintained at $5 \mu \mathrm{l} / \mathrm{min}$ during the

352 entire experiment by an NE-1000 Syringe Pump (New Era Pump Systems, NY). To ensure steady-state

353 growth, the cells were left to grow in channels at least $14 \mathrm{hr}$ before imaging started.

Estimation of mNG maturation time by translational arrest with chloramphenicol. For chloramphenicol treatment extra tubing was connected to microfluidic chips that contained $\mathrm{M} 9$ media with $300 \mu \mathrm{g} / \mathrm{ml}$ chloramphenicol. Care was taken for the drug from the tubing not to reach the cells before treatment by backfilling part of the tubing with the regular medium. Once normal cell growth was verified in regular $\mu \mathrm{l} / \mathrm{min})$. Cells were imaged in the presence of chloramphenicol for $4 \mathrm{hr}$. After protein synthesis stopped as a result of chloramphenicol treatment, $\mathrm{mNG}$ fluorescence continued to increase due to maturation of the fluorophore. An increase of total fluorescence from $\mathrm{mNG}$ as a function of time allows determining the rate constant for maturation $k_{\text {mat }}$ from an exponential fit (SI Fig S2) (50).

Inhibition of FtsZ polymerization by SulA expression. E. coli strain (MB43) expressing pA3 plasmid with the sulA gene under the control of lac-promoter (53) was used in these experiments. IPTG ( $80 \mu \mathrm{M})$, which was used for induction of the SulA, was delivered to cells following the same procedure as described for chloramphenicol.

367 Microscopy. A Nikon Ti-E inverted fluorescence microscope with a 100X NA 1.40 oil immersion phase contrast objective and Perfect Focus system was used for imaging the bacteria in microfluidic channels. Fluorescence was excited by a 200W Hg lamp through ND4 and ND8 neutral density filters. Chroma 41001 filter-cube was used to record mNG and GFP images. Images were captured by an Andor iXon DU897

371 camera and recorded using NIS-Elements software. Power from Hg lamp was measured before time-lapse 372 imaging using Thorlabs PM121D power meter. All strains were recorded at nominally the same 
373 illumination conditions except for the FtsZ-mNG strain in M9 glycerol, which exposure was increased twice

374 due to its lower level of Ftsz.

375 Data analysis. Matlab, along with the Image Analysis Toolbox and Diplmage Toolbox,

376 (http://www.diplib.org/) was used for image analysis. In all analysis of time-lapse recordings, corrections

377 to subpixel shifts between different frames were applied first. These shifts were determined by correlating

378 phase contrast images in adjacent frames. The cells were then segmented based on phase contrast images

379 using a custom Matlab script. The script first identified the coordinates of the two poles of each cell. These

380 coordinates defined the long axes of the cell and yielded cell length. Intensity line profiles along the axes

381 were then determined by integrating parallel line profiles along this centerline and subtracting the

382 background. These profiles were plotted as kymographs for each cell. Segmentation routine allowed for

383 joining daughter cells after their division. The total intensity from the profiles integrated over cell length

384 is proportional to the number of fluorescent FtsZ-mNG in the cell $(\mathrm{N})$. Concentration was calculated by

385 dividing total intensity by the cell length assuming the same widths for all the cells.

386 Piecewise linear fits to total fluorescence curves were carried out using a custom Matlab script. Number

387 of different segments for $\Delta c l p P$ and $\Delta c l p X$ strains was four and for WT strains six. The end point intensities

388 and times for each segment were treated as fitting parameters. The exponential fits to find maturation

389 rate of $m N G$ and linear fits to find basal rate of FtsZ degradation were carried out in Origin 2016 software.

390 Further discussion on accuracy of this procedure is given in SI Modelling.

\section{Acknowledgements}

392 The authors thank Harold Erickson, Jie Xiao and Alex Dajkovic for strains and plasmids, Da Yang and Scott

393 Retterer for help in microfluidic chip making, and Harold Erickson and Maxim Lavrentovich for valuable

394 comments. Authors acknowledge technical assistance and material support from the Center for

395 Environmental Biotechnology at the University of Tennessee. A part of this research was conducted at the 
Scientific User Facilities Division, Office of Basic Energy Sciences, U.S. Department of Energy. This work

has been supported in part by NSF research grant MCB-1252890.

\section{References}

401 1. Willis L \& Huang KC (2017) Sizing up the bacterial cell cycle. Nat. Rev. Microbiol. 15(10):606-620.

402 2. Adams DW, Wu LJ, \& Errington J (2014) Cell cycle regulation by the bacterial nucleoid. Curr. Opin. Microbiol. 22:94-101.

3. Männik J \& Bailey MW (2015) Spatial coordination between chromosomes and cell division proteins in Escherichia coli. Frontiers in Microbiology. 6:306. 10.3389/fmicb.2015.00306.

406

4. Bi E \& Lutkenhaus J (1991) FtsZ ring structure associated with division in Escherichia coli. Nature 354(6349):161-164.

7. Mukherjee A \& Lutkenhaus J (1994) Guanine Nucleotide-Dependent Assembly of Ftsz into

5. Haeusser DP \& Margolin W (2016) Splitsville: structural and functional insights into the dynamic

6. Vaughan S, Wickstead B, Gull K, \& Addinall SG (2004) Molecular evolution of FtsZ protein sequences encoded within the genomes of archaea, bacteria, and eukaryota. J Mol Evol 58(1):1929.

8. Erickson HP, Anderson DE, \& Osawa M (2010) FtsZ in bacterial cytokinesis: cytoskeleton and force Filaments. J. Bacteriol. 176(9):2754-2758.

9. Yang XX, et al. (2017) GTPase activity-coupled treadmilling of the bacterial tubulin FtsZ organizes septal cell wall synthesis. Science 355(6326):744.

10. Bisson AW, et al. (2017) Treadmilling by FtsZ filaments drives peptidoglycan synthesis and bacterial cell division. Science 355(6326):739-743.

11. Loose M \& Mitchison TJ (2014) The bacterial cell division proteins FtsA and FtsZ self-organize into dynamic cytoskeletal patterns. Nat. Cell Biol. 16(1):38.

12. Mingorance J, Rivas G, Velez M, Gomez-Puertas P, \& Vicente M (2010) Strong FtsZ is with the force: mechanisms to constrict bacteria. Trends in Microbiology 18(8):348-356.

13. Popp D, Iwasa M, Narita A, Erickson HP, \& Maeda Y (2009) FtsZ Condensates: An In Vitro Electron Microscopy Study. Biopolymers 91(5):340-350.

14. Szwedziak P, Wang Q, Bharat TAM, Tsim M, \& Lowe J (2014) Architecture of the ring formed by the tubulin homologue FtsZ in bacterial cell division. Elife. 3. 10.7554/eLife.04601.

15. Addinall SG \& Lutkenhaus J (1996) FtsA is localized to the septum in an FtsZ-dependent manner. J Bacteriol 178(24):7167-7172. 
16. Hale CA \& deBoer PAJ (1997) Direct binding of FtsZ to ZipA, an essential component of the septal ring structure that mediates cell division in E. coli. Cell 88(2):175-185.

17. Aarsman MEG, et al. (2005) Maturation of the Escherichia coli divisome occurs in two steps. Mol. Microbiol. 55(6):1631-1645.

18. Liu B, Persons L, Lee L, \& de Boer PAJ (2015) Roles for both FtsA and the FtsBLQ subcomplex in FtsN-stimulated cell constriction in Escherichia coli. Mol. Microbiol. 95(6):945-970.

19. de Boer PAJ (2010) Advances in understanding E. coli cell fission. Curr. Opin. Microbiol. 13(6):730737.

20. Typas A, Banzhaf M, Gross CA, \& Vollmer W (2012) From the regulation of peptidoglycan synthesis to bacterial growth and morphology. Nat. Rev. Microbiol. 10(2):123-136.

21. Stouf M, Meile J-C, \& Cornet F (2013) FtsK actively segregates sister chromosomes in Escherichia coli. Proc. Natl. Acad. Sci. U. S. A. 110(27):11157-11162.

22. Männik J, Bailey MW, O'Neill JC, \& Männik J (2017) Kinetics of large-scale chromosomal movement during asymmetric cell division in Escherichia coli. PLoS Genet. 13(2):e1006638.

23. Adams DW \& Errington J (2009) Bacterial cell division: assembly, maintenance and disassembly of the $\mathrm{Z}$ ring. Nat. Rev. Microbiol. 7(9):642-653.

24. Rueda S, Vicente M, \& Mingorance J (2003) Concentration and assembly of the division ring proteins FtsZ, FtsA, and ZipA during the Escherichia coli cell cycle. J Bacteriol 185(11):3344-3351.

25. Weart RB \& Levin PA (2003) Growth rate-dependent regulation of medial FtsZ ring formation. J Bacteriol 185(9):2826-2834.

26. Romberg L \& Levin PA (2003) Assembly dynamics of the bacterial cell division protein FTSZ: poised at the edge of stability. Annu Rev Microbiol 57:125-154.

27. Moriya S, Rashid RA, Rodrigues CDA, \& Harry EJ (2010) Influence of the nucleoid and the early stages of DNA replication on positioning the division site in Bacillus subtilis. Mol. Microbiol. 76(3):634-647.

28. Bernhardt TG \& de Boer PAJ (2005) SImA, a nucleoid-associated, FtsZ binding protein required for blocking septal ring assembly over chromosomes in E. coli. Mol. Cell 18(5):555-564.

29. Bailey MW, Bissichia P, Warren BT, Sherratt DJ, \& Männik J (2014) Evidence for divisome localization mechanisms independent of the Min system and SImA in Escherichia coli. PLoS Genet. 10(8):e1004504. 10.1371/journal.pgen.1004504.

30. Wu LJ \& Errington J (2004) Coordination of cell division and chromosome segregation by a nucleoid occlusion protein in Bacillus subtillis. Cell 117(7):915-925.

31. Dewachter L, Verstraeten N, Fauvart M, \& Michiels J (2018) An integrative view of cell cycle control in Escherichia coli. FEMS Microbiol Rev 42(2):116-136.

32. Zhou P \& Helmstetter CE (1994) Relationship between ftsZ gene expression and chromosome replication in Escherichia coli. J Bacteriol 176(19):6100-6106.

33. Garrido T, Sanchez M, Palacios P, Aldea M, \& Vicente M (1993) Transcription of ftsZ oscillates during the cell cycle of Escherichia coli. EMBO J 12(10):3957-3965.

34. Dewar SJ, Kagan-Zur V, Begg KJ, \& Donachie WD (1989) Transcriptional regulation of cell division genes in Escherichia coli. Mol Microbiol 3(10):1371-1377. 
471 35. Robin A, Joseleau-Petit D, \& D'Ari R (1990) Transcription of the ftsZ gene and cell division in

36. Quardokus E, Din N, \& Brun YV (1996) Cell cycle regulation and cell type-specific localization of

37. Holtzendorff J, et al. (2001) Diel expression of cell cycle-related genes in synchronized cultures of

38. Mori T \& Johnson CH (2001) Independence of circadian timing from cell division in cyanobacteria. J Bacteriol 183(8):2439-2444.

39. Piir K, Paier A, Liiv A, Tenson T, \& Maivali U (2011) Ribosome degradation in growing bacteria.

40. Vischer NOE, et al. (2015) Cell age dependent concentration of Escherichia coli divisome proteins

41. Moore DA, Whatley ZN, Joshi CP, Osawa M, \& Erickson HP (2017) Probing for Binding Regions of

42. Wang P, et al. (2010) Robust Growth of Escherichia coli. Curr. Biol. 20(12):1099-1103.

43. Yang D, Jennings AD, Borrego E, Retterer ST, \& Männik J (2018) Analysis of Factors Limiting

44. Kitagawa M, et al. (2005) Complete set of ORF clones of Escherichia coli ASKA library (A complete Bacterial Growth in PDMS Mother Machine Devices. Frontiers in Microbiology. 9(871). 10.3389/fmicb.2018.00871.

45. Flynn JM, Neher SB, Kim YI, Sauer RT, \& Baker TA (2003) Proteomic discovery of cellular substrates

46. Camberg JL, Hoskins JR, \& Wickner S (2009) ClpXP protease degrades the cytoskeletal protein,

47. Olivares AO, Baker TA, \& Sauer RT (2018) Mechanical Protein Unfolding and Degradation. Annu

48. Camberg JL, Viola MG, Rea L, Hoskins JR, \& Wickner S (2014) Location of Dual Sites in E. coli FtsZ

49. Gottesman S, Roche E, Zhou Y, \& Sauer RT (1998) The ClpXP and ClpAP proteases degrade proteins

50. Balleza E, Kim JM, \& Cluzel P (2018) Systematic characterization of maturation time of fluorescent

51. Elowitz MB, Levine AJ, Siggia ED, \& Swain PS (2002) Stochastic gene expression in a single cell.

52. Maillard RA, et al. (2011) ClpX(P) Generates Mechanical Force to Unfold and Translocate Its Protein Substrates. Cell 145(3):459-469. 
511 53. Dajkovic A, Mukherjee A, \& Lutkenhaus J (2008) Investigation of regulation of FtsZ assembly by 512 SulA and development of a model for FtsZ polymerization. J. Bacteriol. 190(7):2513-2526.

513 54. Sun Q \& Margolin W (1998) FtsZ dynamics during the division cycle of live Escherichia coli cells. J. 514 Bacteriol. 180(8):2050-2056.

515 55. Golding I, Paulsson J, Zawilski SM, \& Cox EC (2005) Real-time kinetics of gene activity in individual 516 bacteria. Cell 123(6):1025-1036.

517 56. Kelly AJ, Sackett MJ, Din N, Quardokus E, \& Brun YV (1998) Cell cycle-dependent transcriptional 518 and proteolytic regulation of FtsZ in Caulobacter. Genes Dev 12(6):880-893.

519 57. Williams B, Bhat N, Chien P, \& Shapiro L (2014) ClpXP and ClpAP proteolytic activity on divisome 520 substrates is differentially regulated following the Caulobacter asymmetric cell division. Mol 521 Microbiol 93(5):853-866.

522

58. Bates D \& Kleckner N (2005) Chromosome and replisome dynamics in E. coli: Loss of sister cohesion triggers global chromosome movement and mediates chromosome segregation. Cell 121(6):899-911.

526

59. Collier J (2016) Cell cycle control in Alphaproteobacteria. Curr Opin Microbiol 30:107-113.

60. Dewar SJ \& Dorazi R (2000) Control of division gene expression in Escherichia coli. FEMS Microbiol

61. Pazos M, Natale P, \& Vicente M (2013) A specific role for the ZipA protein in cell division: stabilization of the FtsZ protein. J Biol Chem 288(5):3219-3226.

530

62. LaBreck CJ, May S, Viola MG, Conti J, \& Camberg JL (2017) The Protein Chaperone ClpX Targets 


\section{Figures}
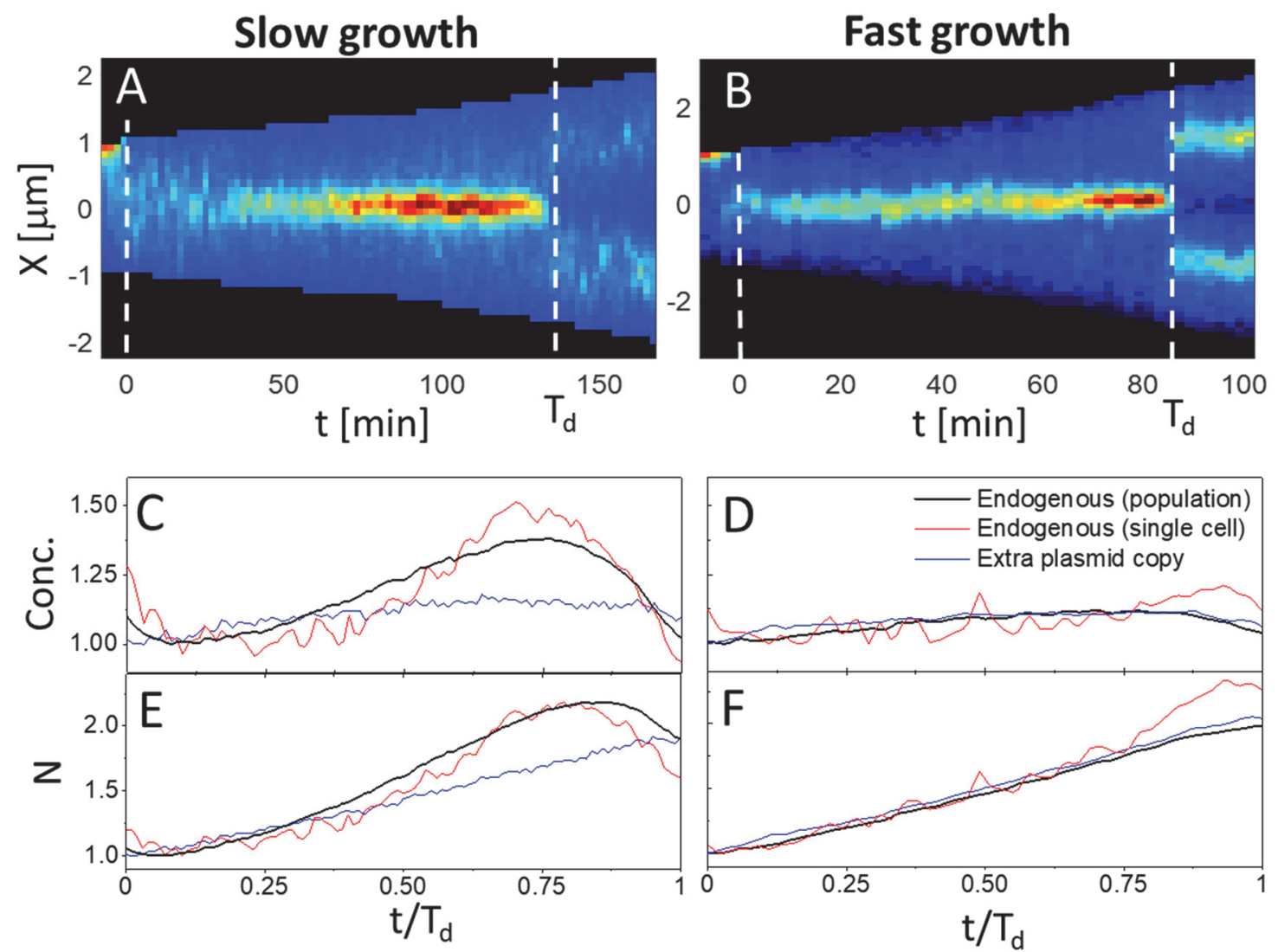

Figure 1. Changes in fluorescent FtsZ concentrations and numbers during cell cycle. Left column corresponds to slow and right column to fast growth conditions. (A, B) Kymographs of FtsZ-mNG intensity distribution along the long axes in representative cells. Red corresponds to high concentration and blue to low. Black marks areas outside the cell. Vertical dashed lines mark times of cell division. (C)

539 Concentration of fluorescent FtsZ construct as a function of cell cycle time in slow growth conditions. Thick 540 black line corresponds to the population average data from the strain expressing a sole FtsZ-mNG from 541 the native locus $(\mathrm{N}=53)$, thin blue line to the strain where FtsZ-GFP is expressed from a plasmid in addition 542 to native unlabeled copy ( $\mathrm{N}=16)$ and thin red line to the cell shown on the kymograph above. (D) The same 543 for fast growth conditions. $\mathrm{N}=32$ for endogenous and $\mathrm{N}=33$ for plasmid expressing strain. (E, F) Numbers 544 of fluorescent FtsZ in the cell in slow and fast growth conditions, respectively. All curves in panels (C)-(F) are normalized by the cell cycle minimum value. 


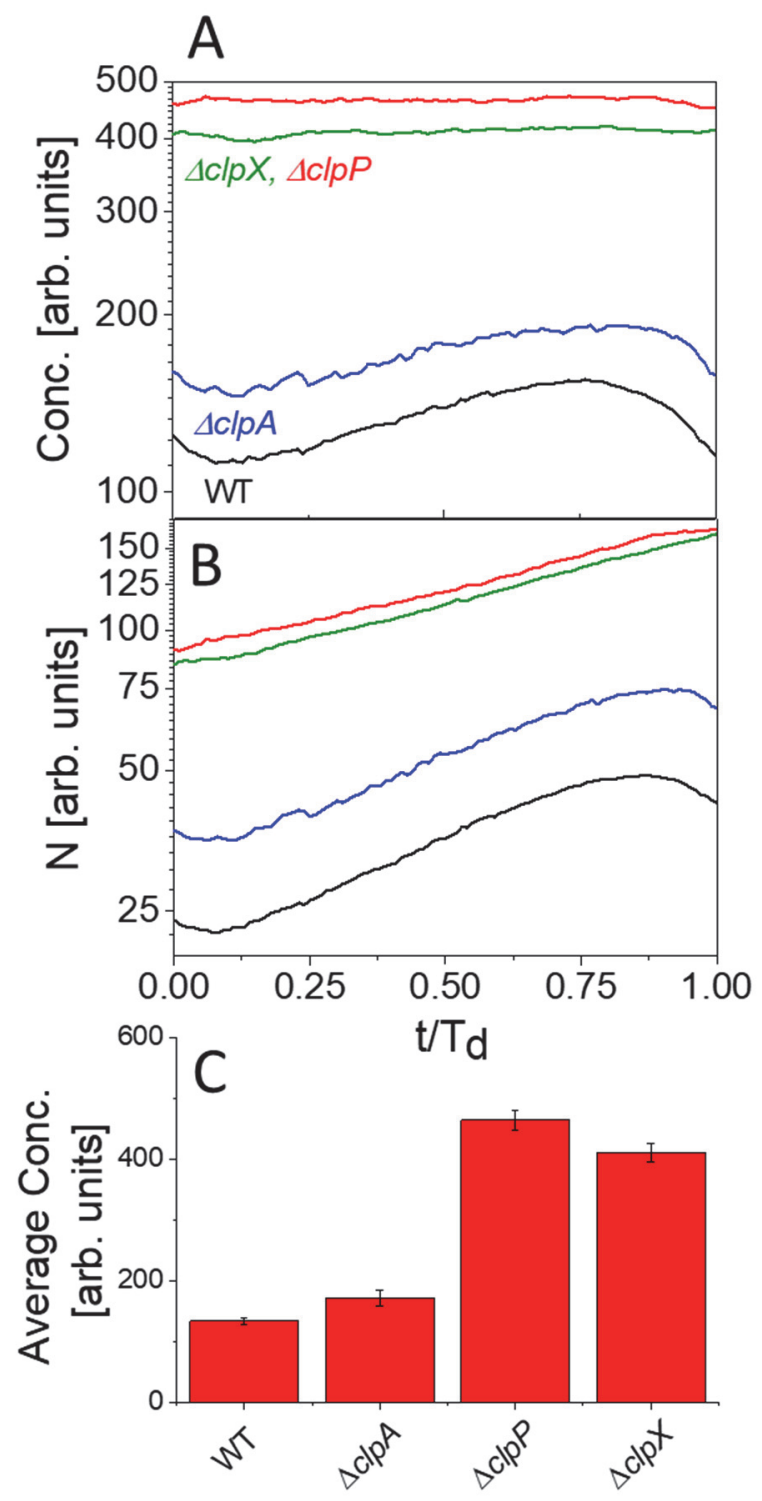

547 Figure 2. Comparison of FtsZ abundances and concentrations in WT and the protease deletion strains. (A)

548 Population average concentration of FtsZ-mNG as a function of cell cycle time for WT (N=53), $\Delta c / p A$

$549(\mathrm{~N}=16), \Delta c / p X(\mathrm{~N}=31)$ and $\Delta c / p P(\mathrm{~N}=36)$ strains. All strains have been measured in slow growth conditions

550 and express FtsZ-mNG from the native locus. (B) Total number of fluorescent FtsZ-mNG in these strains

551 as a function of cell cycle time. Vertical axes in panels (A) and (B) is logarithmic. (C) Concentration of

552 fluorescent FtsZ-mNG averaged over cell cycle in different strains. Error bars are s.e.m. 

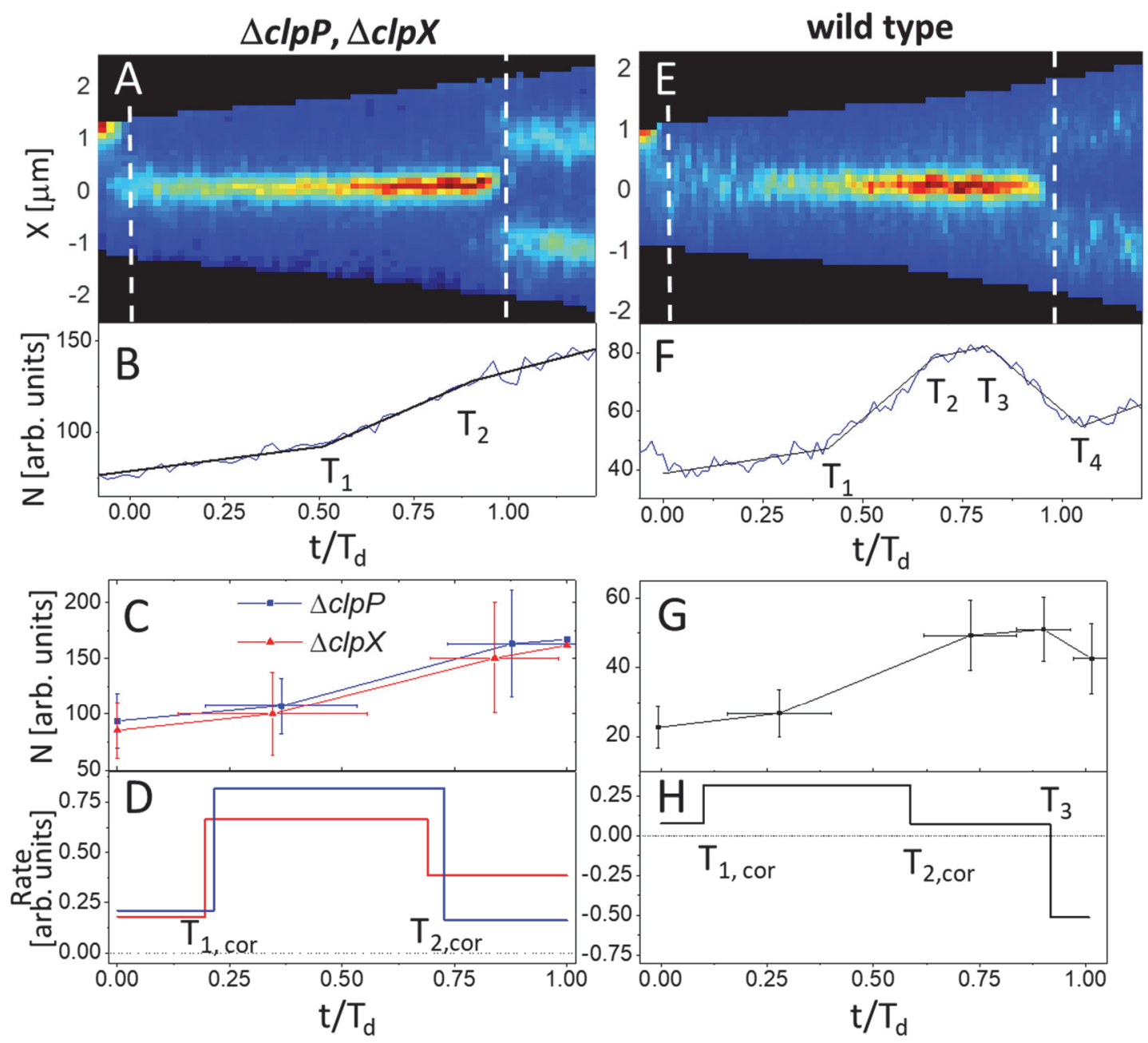

554 Figure 3. Rates of change in FtsZ numbers during cell cycle in ClpX and ClpP null mutant (left column) and 555 WT (right column) strains in slow growth conditions. (A, E) Kymographs of FtsZ-mNG distribution along the long axes in representative cells. Panel (A) shows a $\triangle c l p P$ cell. Vertical dashed lines mark cell divisions. (B, F) Number of fluorescent FtsZ-mNG as a function of the cell cycle time for the cells shown above. Solid 558 black lines correspond to piecewise linear fit. $T_{1}-T_{4}$ mark times when the slope of the curve changes. $T_{1}$ upregulation of FtsZ synthesis; $T_{2}$ - slow-down of the FtsZ synthesis; $T_{3}$ - start of rapid FtsZ degradation, and $\mathrm{T}_{4}$ - end of rapid FtsZ degradation. $(C, G)$ The fitting curves after averaging them over cell populations.

$561 \mathrm{~N}=35$ for $\Delta c l p P, \mathrm{~N}=32$ for $\Delta c l p X$ and $\mathrm{N}=53$ for WT cells. Error bars are s. d. (D, H) rates of change in FtsZ$562 \mathrm{mNG}$ numbers. The rates correspond to the slopes of the curves from the panel above. Times $\mathrm{T}_{1, \text { cor }}$ and $563 \mathrm{~T}_{2, \text { cor }}$ have been corrected to account for the maturation of $\mathrm{mNG}$. 

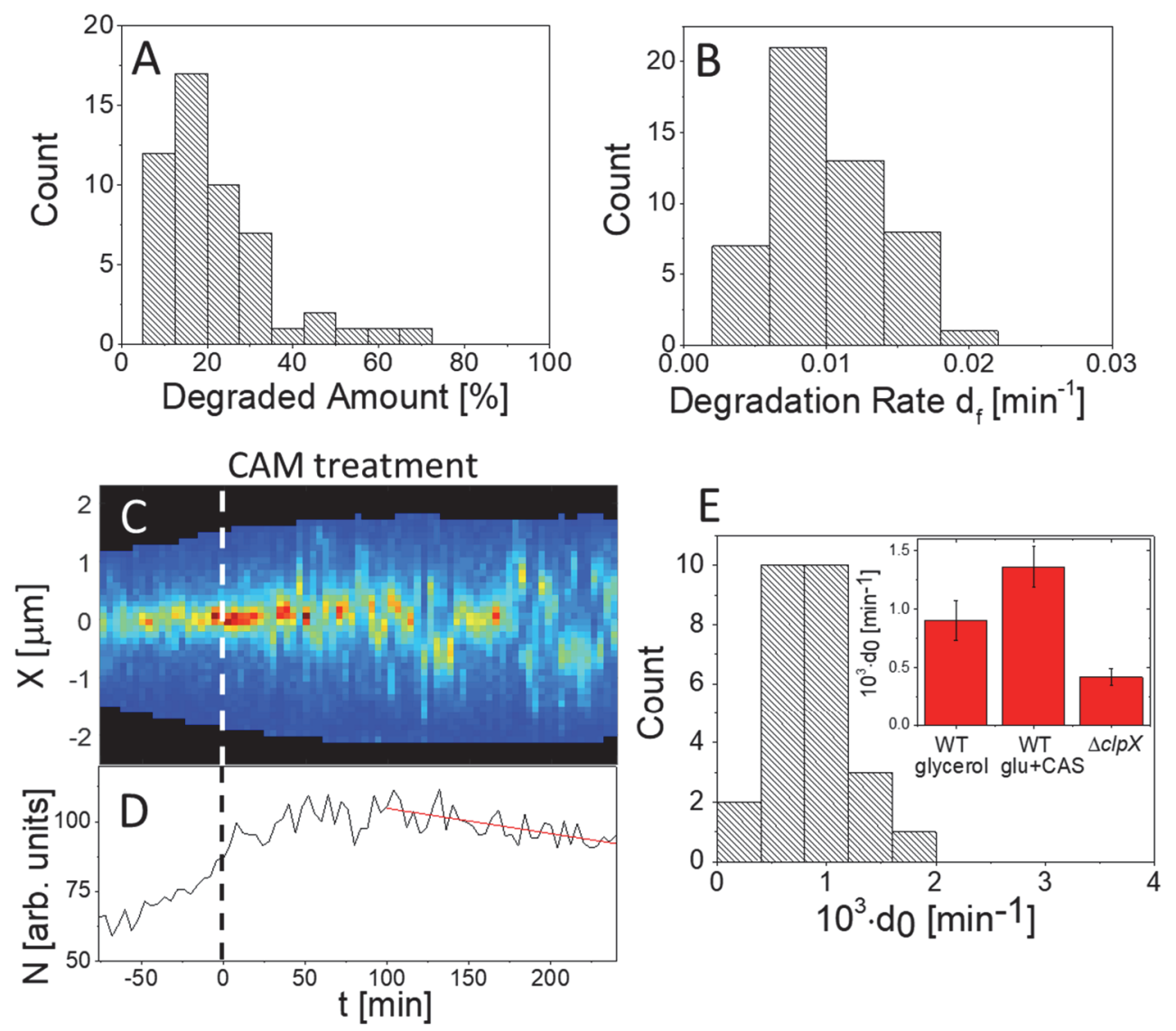

Figure 4. Degradation rates of FtsZ in the beginning and end of the cell cycle. (A) Degraded amount of FtsZ in the end of the cell cycle at the time when FtsZ disassociates from the Z-ring. The amount is relative to the amount at the end of cell division. (B) Degradation rate during this period. $N=53$. (C) Kymograph showing FtsZ-mNG distribution along the long axes of the cell before and during chloramphenicol treatment (CAM, $300 \mu \mathrm{g} / \mathrm{ml}$ ). Drug is administered at time 0 min (vertical dashed line). (D) Amount of

570 fluorescent FtsZ-mNG in this cell as a function of time. Basal degradation rate, $d_{0}$, is determined from the 571 linear fit to the curve (red solid line). (E) Distribution of $d_{0}$ in WT cell population in slow growth conditions $572(\mathrm{~N}=26)$. Inset shows the population average basal rate for WT cells in slow and fast growth conditions and 573 for $\Delta c l p X$ cells. The latter acts as a control. Error bars are s. $\mathrm{d}$. 

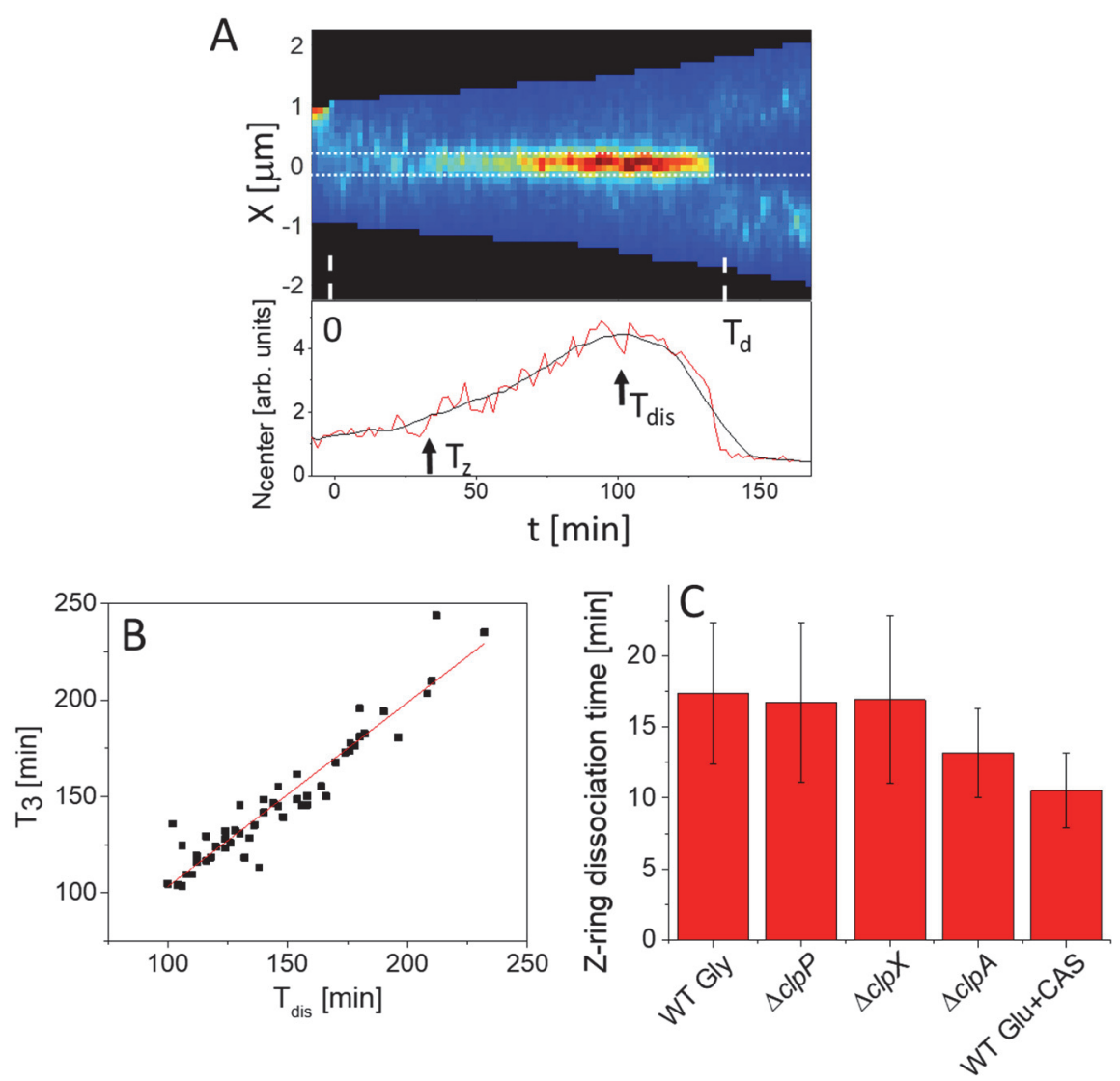

576 Figure 5. Rapid degradation of FtsZ is a consequence of the Z-ring dissociation. (A) Kymograph (top) and

577 amount of fluorescent FtsZ-mNG at the cell center (bottom) in a representative cell. The cell center is

578 defined as $300 \mathrm{~nm}$ wide band (indicated by horizontal dotted lines on kymograph). Fluorescent intensity

579 after averaging by a running window is shown by a black line. $T_{2}$ indicates start time and $T_{\text {dis }}$ time when

580 the Z-ring starts to dissociate. (B) Start time for FtsZ dissociation from constriction region ( $T_{\text {dis }}$ ) vs start

581 time of rapid degradation of FtsZ $\left(T_{3}\right)$. (C) Total time for FtsZ to dissociate from the Z-ring $\left(T_{d}-T_{d i s}\right)$ in

582 different strains and growth conditions. Error bars are s. d. 

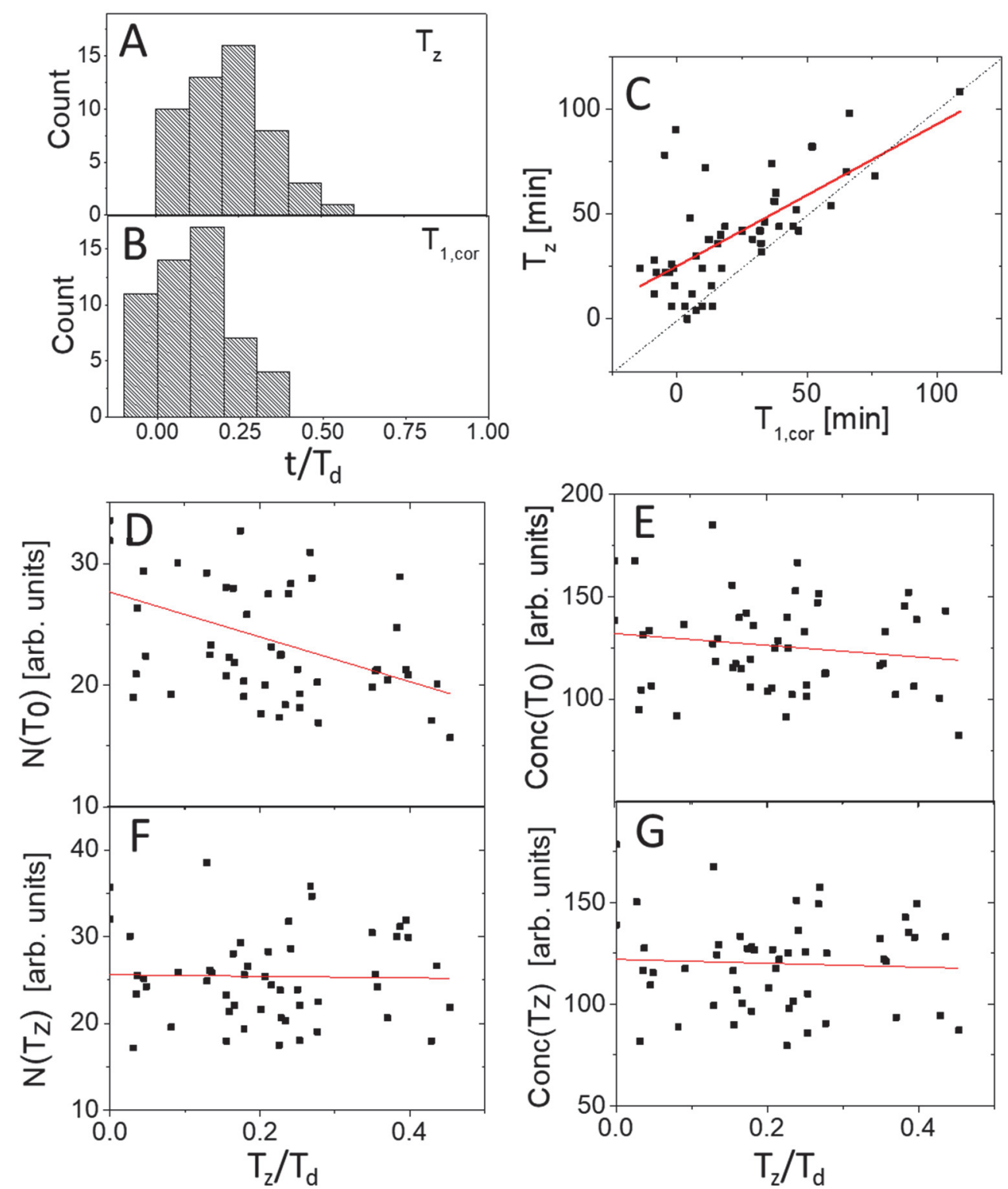

584 Figure 6. Link between upregulation of FtsZ synthesis and formation of the Z-ring in slow growth conditions. (A) Distribution of times when Z-ring forms $\left(T_{z}\right)$. (B) Distribution of times when FtsZ synthesis rate increases during the cell cycle $\left(T_{1, \text { cor }}\right)$. The times are corrected for mNG maturation time. (C) $T_{2}$ vs $T_{1, \text { cor }}$

587 for the same dataset. Dashed black line corresponds to $T_{2}=T_{1, \text { cor }}$ and solid red line to linear fit $\left(T_{z}=\right.$ $\left.588-0.67 T_{1, \text { cor }}+25.2 ; R=-0.67\right)$. (D) Number of fluorescent FtsZ-mNG and (E) its concentration at cell birth $589\left(T_{0}\right)$ vs. time when Z-ring forms. (F) Number of fluorescent FtsZ-mNG and (G) its concentration at the time 590 of Z-ring formation vs. time when Z-ring forms. Solid lines are fits to the data. The fitting parameters are 591 listed in SI Table S2. N=53. 


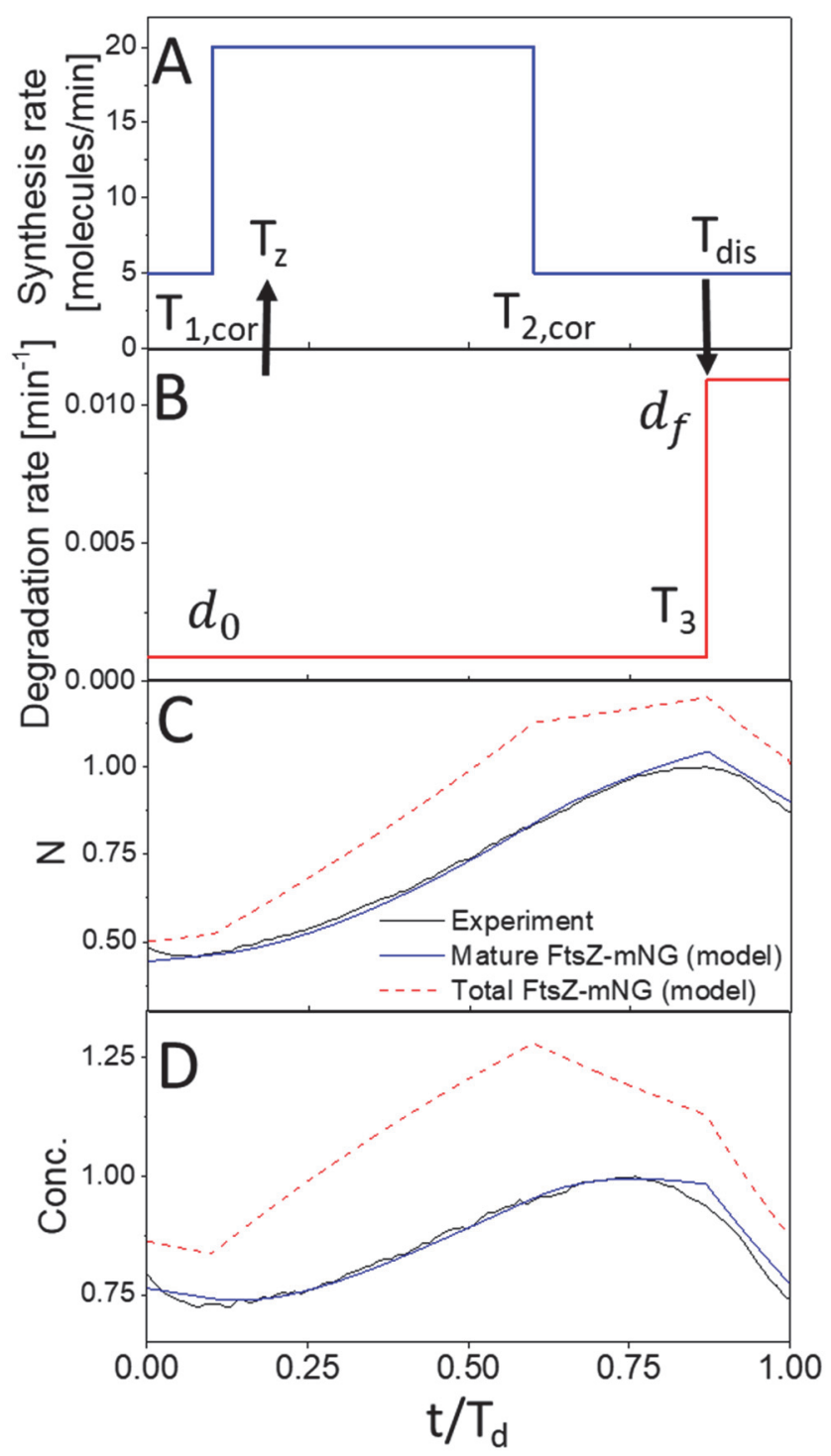

593 Figure 7. Comparing model and experiment. (A) Synthesis rate as function of time used in the model. The 594 rate is 4 times the basal rate between $T_{1, \text { cor }}=0.1 T_{d}$ and $T_{2, \text { cor }}=0.6 T_{d}$ in accordance with the measurements.

595 (B) Degradation rate as a function of time. Degradation rate increases from $0.001 \mathrm{~min}^{-1}$ to $0.010 \mathrm{~min}^{-1}$ at $5960.9 \mathrm{~T}_{\mathrm{d}}$. (C) Comparison of number of FtsZ molecules in the cell. The numbers are normalized by the 597 maximum number of mature FtsZ-mNG during the cell cycle. Solid black line corresponds to the 598 experiment, blue line to mature, and dashed red line total FtsZ-mNG from the model. The additional 599 model parameters not specified on panels $(A)$ and $(B)$ are $T_{d}=160 \mathrm{~min}$ and maturation rate $k_{\text {mat }}=0.027$ $600 \mathrm{~min}^{-1}$. (D) Comparison of FtsZ concentrations. The concentrations have been normalized by maximum 601 concentration of FtsZ-mNG during the cell cycle. 